As the disease is said to be assoclated with changes in the pituitary body, it is just possible that the early fracture of the frontal bone may have had some bearing on the local condition elther by reflexly damaging that organ or by causing some obscure changes in its growth.

The two photographs and tracing of the toes of the left foot will give a fair idea of his local condition.

\section{ADDISON'S DISEASE}

By WILLIAM TIBBLES, LL.D., L.R C.P., M.R.C.S., Etc. Nottingham.

Among the various discolorations of the skin Addison's disease holds an important place, on account of the constitutional changes which precede and accompany it, its frequently fatal termination, and its association with disesse of the suprarenal bodies. Although the functions of these organs are not thoroughly understood, we may include amongst them (a) the elaboration of a secretion which is essential to the blood (haemopoietic), (b) the destruction of effete products of metabolism, especially haemoglobin, (c) and nervous functions which are closely associated with those of the great sympathetic system of the abdomen.

While excesslve or irregular pigmentation of the skin is one of the most striking phenomena of Addison's disease, this can only be regarded as a cutaneous manifestation of a deep-seated malady involving the mechanism which produces or governs the distribution of pigment in the body. In the early stage, probably before any irregular or excessive pig mentation is observed, there is a gradually-increasin debility, breathlessness on $\epsilon$ xertion, a rapid, feeble pulse, and weak heart sounds, and epigastric or hypochondriac pain which may be mistaken for dyspepsia or liver trouble; as the disease advances there are added to these giddiness, faintness, or a tendency to fall on rising from a bed or couch, and other cerebral troubles; the epigastric or hypochondriac pain persists, and anorexia, nausea, vomiting, and occasional diarrhoea are common; the nutrition is maintained, at any rate emaciation is not usual, and the temperature remains normal or subnormal.

Many of these symptoms give to the disease a complexion similar to various forms of auto-intoxication; and as the suprarenals are depurative organs, it is probable that the increasing debility, giddiness, cloudiness of the intellect, nansea, vomiting, diarrhoea, and similar symptoms are due to poisoning by bodies (probably leucomaines) resulting from the destruction of red corpuscles and the staining of the skin to the circulation of the products of disintegration of haemoglobin. However, in many cases of Addison's disease, the tuberculous or malignant process which is going on in the suprarenal bodies is accompanied by degenerative changes in the semilunar ganglia and branches, of the solar plexus; such a disturbance of the great splanchnic nervous system could not occur without corresponding subjective changes, such as indigestion, nausea, vomiting, diarrhoea, and disturbance of the heart's action. It seems probable, therefore, that dis turbance of the sympathetic nervous system and autointoxication are both concerned in these results, while pro gressive caseation or spread of malignant infection, when such occur, hasten the fatal termination.

CASE I

W. B. was a man of middle age when I first knew him, and he lived five years. He had very dark skin, hair, and eyebrows, and dark brown eyes; was born in the tropios of English parents; was stout, short of breath, and had occasional attacks of bronchitis. His occupation cause him to attend many public meetings, and led to the development of oratorical powers; buc in 1895 he complained that his mental power was failing him; his words did not flow so freely as was their wont sometimes he lost the thread of his discourse or was fast in the middle of a sentence for a word to express his meaning, and he was then unable to go on for a few seconds; the loss of memory was accompanied by giddiness and dimness of vision-sverything was blank. When matter had reached this stage the pigment in his skin was much increased, and arranged in irregular but well-defined patches upon his back chest, abdomen, and extremities ; the linea alba was much pigmented and the sorotum nearly black; his sclerotics and the lunulae on his finger-nails were free from pigment, but there were dark spots on the mucous membrane of his mouth and tongue, a dark line on the lip near the junction of skin and mucous membrane, and dark lines in the palms of the hands in the natural creases of the skin : his digestion was disturbed, appetite very bad, vomiting occasionally, aching pain and tenderness in the hypochondriac and epigastric regions; his breathing and pulse were rapid and temperature subnormal. On a certain dag the vomiting was so severe as to bring up much blood. From this time his condition became rapidly worse and he had to relinguish his duties; in a few weeks his circulatory and musoular powers were sn cnfeebled that he could with difficulty walk upstairs or about his room ; anasarca, beginning in his feet and ankles, soon becrme general, and he was confined to a chair or bed; his once bright intellect became clouded, his powers of thought, concentration, and memory faded, and he sank from exhaustion about eighteen months after the diagnosis of his case.

The special points in this case were the colour of the ekin, the freedom of the sclerotics and roots of the nails from pigment and the urine from bile, the absence of signs of liver or other disease which causes pigmentation, the appearance of the spots on the mucous membrane of the mouth and tongue, and of lines on the lips and palms of the hands, and the constitutional disturbance, and these agree with a diagnosis of Addison's disease.

\section{Case II.}

B. M., aged 30 , came complaining of a dull, aching pain, and feeling of fullness in the left hypochondriac region; shortness of breath, increasing languor and debility, drowsiness, giddiness and sickness on rising from bed, palpitation on exertion, poor appetite, constipation. Her menses were regular; pulso, 120 ; temperature, $98^{\circ} \mathrm{F}$. In appearance she was fairly well-nourighed, and a brunette, black hair and eyebrows, brown eyes. There was considerable bronzing of her skin, but it was not uniform, and showed itself chiefly as large patches on the back. chest, and abdomen, which shaded off into normal or fair skin; the back of the forearms and hands, and the entire flngers were of a dark greenish-brown colour, as though they were stained with dye; upon the chin, the dorsal surface of each hand and of the right forearm were patches of skin, several inches in diameter, which had a white and shining appearance, smooth and colourless, but soft, pliant, level with the general surface, and having normal sensation. The pigmentation, and consequently the contrast of the white patches, was much increased by several weeks at the seaside.

The disease began two years before in an insidious manner; the first signs of ill-health being shortness of breath and debility, but as the pain increased the pigmented condition of her skin became more marked and the constitutional signs were severe ; Addison's disease was diagnosed. The gradual development of the white patches was puzzling; there was no history of injury or neuritis to account for them, nor was there any burning pain or pinkiness of the skin as described by Paget in atrophodermia neuritica; nor were the patches due to atrophodermia pigmentosa, for, although the whiteness followed the pigmentation, as in that malady, they did not begin in childhood, but were comparatively recent; nor had they a depressed and shrunken appearance or pink spots of dilated capillaries upon them; on the contrary, they were soft, pliant, movable, could be pinched up with the fingers, and it was concluded that they were patches of leucodermia occurring in the course of Addison's disease.

She was placed under a course of treatment in which the principal drugs used were iron, arsenic, and nux vomica, for six months. During this time the pigmentation became noticeably less, the white patches lost their shiny appearance and approximated somewhat to a normal colour ; the giddiness, sickness, palpitation also diminished, and the patient declared herself better than she had been for years, but whether and how soon a relapse will occur one cannot hazard a guess.

I have seen several other cases in which disturbance of pigmentation in persons of neurotic temperament has been improved by similar treatment; and it seems probable, in all cases which show marked improvement by treatment, that the nervous elements of the suprarenals have been principally involved, and that a marked degree of degeneration cannot have taken place. It is possible, however, that certain tuberculous changes may take place in these organs without a fatal result following, that such a process inay end in caseation or calcification as elsewhere. The degenerated elements can never be restored, but, the process being checked, the body may accustom itself to the absence of some portion of adrenal secretion, or the healthy organ of the opposite side may in time become more active and supply the needed material.

\section{THE PARATHYROIDS IN GRAVES'S DISEASE.}

By S. G. SHATTOCK, F.R.C.S.,

Pathological Curator, Royal College of Burgeons of England, etc.

Tre publication by Dr. Laurence Hamphry in the Lancet, November I1th, 1905, p. 1390, of his observations upon the above subject, leads me to publish the following description of a specimen of exophthalmic goitre which was added to the Museam of the Royal College of Surgeons, London, in Julg 